\title{
RELACIONES CERÁMICAS Y SOCIALES ENTRE SAN PEDRO DE ATACAMA Y EL LOA SUPERIOR DURANTE EL PERÍODO INTERMEDIO TARDÍO A TRAVÉS DEL ANÁLISIS DE FLUORESCENCIA DE RAYOS X PORTÁTIL
}

\author{
Emily Stovel, ${ }^{1}$ William Whitehead, ${ }^{2}$ Michael Deibel ${ }^{3}$ y Mauricio Uribe ${ }^{4}$
}

* Introducción Durante el período Intermedio Tardío las comunidades de San Pedro de Atacama y del Loa Superior produjeron tipos cerámicos similares. La cerámica, junto a otros materiales, sugieren una intensificación de una identidad regional compartida durante este período. El análisis de fluorescencia de rayos $\mathrm{X}$ con equipo portátil de 251 fragmentos de cerámica (tipos Dupont, Ayquina y variantes Turi) de varios sitios del Intermedio Tardío indicó que estos tipos cerámicos forman dos grupos químicos distintos vinculados a las dos regiones. Poca cantidad de fragmentos con valores químicos del Loa Superior fueron identificados en la muestra de San Pedro, y ninguno con los valores de San Pedro se encontró en la del Loa Superior. Lo anterior sugiere que en ambas zonas se usaron materias primas propias y locales para coproducir los principales estilos, señalando la existencia de una identidad supraregional. Estos resultados discuten el modelo de un poder diferencial durante el período Tardío para explicar estos patrones.

Palabras claves: San Pedro de Atacama - Loa Superior - fluorescencia de rayos $\mathrm{X}$ portátil - período Intermedio Tardío - cerámica - arqueometría.

During the Late Intermediate Period, communities in San Pedract

Atacama and the neighboring Upper Loa Valley produced similar ceramic styles. Ceramics in combination with other objects, provide evidence of the intensification of a regional identity during this period.

The results of portable X-Ray Fluorescence analysis on 251 ceramic sherds (styles Dupont, Ayquina, and Turi variants) from some sites, shows that these styles form two distinct chemical groups linked to the two areas. A small subset of sherds with chemical values found in the Upper Loa were identified in the San Pedro sample, but none with San Pedro values were found in the Upper Loa sample. This suggests both areas used local raw material resources to coproduce key styles and may have been thereby signaling adherence to a shared regional identity. These results argue against a model of a power differential occurring in the Late Period in explanation of these patterns.

Key words: San Pedro de Atacama - Upper Loa River Valley - portable XRay Fluorescence - Late Intermediate Period - pottery - archaeometry.

Recibido: julio 2012. Aceptado: junio 2013.
Las comunidades de San Pedro de Atacama ocuparon y ocupan una serie de oasis emplazados entre los ríos Vilama y San Pedro, al norte del Salar de Atacama, ubicado a $2450 \mathrm{~m} . \mathrm{snm}$ al pie de los Andes (Figura 1). La región del Loa Superior incorpora una serie de subregiones correspondientes al Alto Loa, el río Salado y el San Pedro, ubicados a partir de los 2500 m.snm. Ambos lugares han sido tema de mucha investigación arqueológica, tanto por el nivel de preservación de sus materiales orgánicos como por su ubicación en el centro de una red de interacción que caracterizó la región por más de 2000 años. La subregión de San Pedro de Atacama ha jugado un importante rol en la definición del Estado prehispánico de Tiwanaku (Berenguer 1978, 1998; Berenguer et al. 1980; Berenguer y Dauelsberg 1989; Oakland 1992; Uribe y Agüero 2001, 2004; Torres-Rouff 2002; Costa et al. 2004; Lechtman y MacFarlane 2005; Knudson 2007); mientras que el río Salado ha sido clave en la exploración de la presencia inca en el norte de Chile (Castro 1992; Gallardo et al. 1995; Varela 1999; Uribe et al. 2002; Uribe 2004; Adán y Uribe 2005; Berenguer et al. 2005).

Por su parte, el período Intermedio Tardío o de los Desarrollos Regionales se ha caracterizado por cambios medioambientales, actividades de minería y metalurgia, complejidad social (como en momentos anteriores y posteriores), la construcción de sitios "defensivos" (como los pucaras de Quitor y Lasana), por un posible aumento en violencia interpersonal y el desarrollo de una identi-

\footnotetext{
1 Instituto de Investigaciones Arqueológicas y Museo Gustavo Le Paige, Universidad Católica del Norte. Gustavo Le Paige 380 , San Pedro de Atacama, CHILE. Email: estovel@ucn.cl
} 


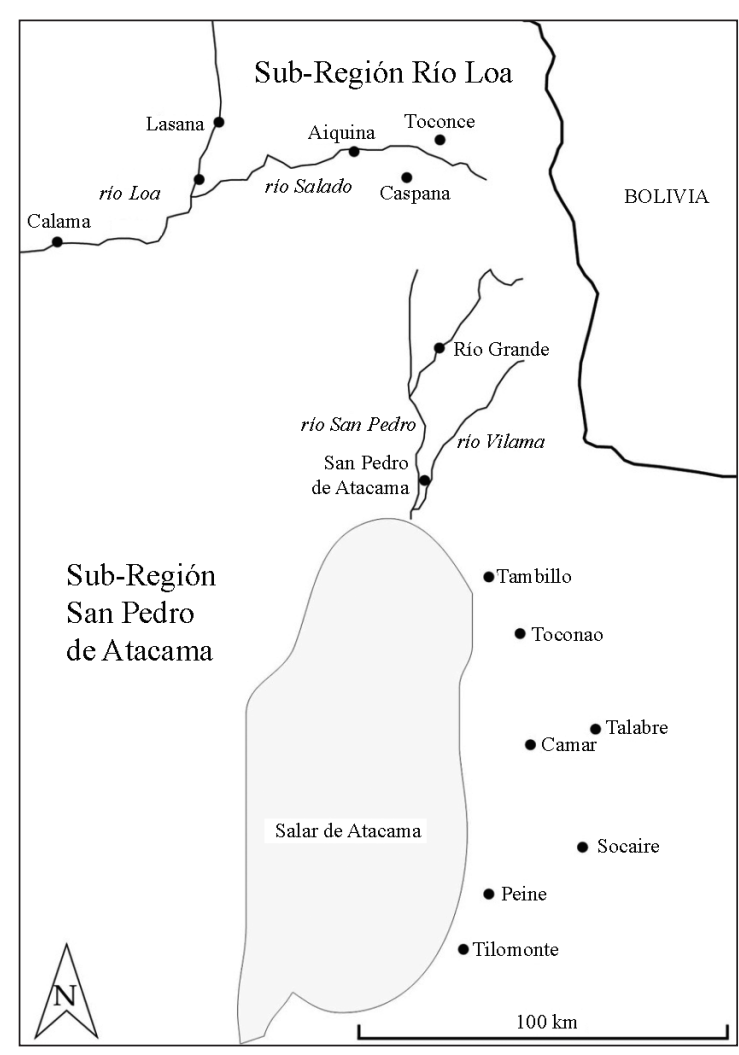

Figura 1. Las subregiones del Loa Superior y San Pedro de Atacama.

dad "atacameña" unificada reflejada por la producción de textiles, cerámica y deformación craneana (Castro et al. 1991; Agüero et al. 1999; Carrasco et al. 2003; Núñez et al. 2003; Torres-Rouff et al. 2005; Torres-Rouff 2007; Sepúlveda 2011). Esta identidad atacameña habría incluido miembros de las comunidades del río Salado y San Pedro de Atacama que produjeron las cerámicas diagnósticas del período. Este conjunto estilístico se ha denominado componente Loa-San Pedro y fue definido por Uribe (1997, 2002), siguiendo clasificaciones cerámicas anteriores (Varela et al. 1991), basadas en prospecciones, excavaciones y estudios de colecciones de ambas zonas (p.e.,

2 University of Wisconsin - Fond du Lac, Department of Anthropology and Sociology, 400 University Drive, Fond du Lac, WI, EEUU. Email: william.whitehead@uwc.edu.

3 Department of Chemistry, Earlham College, 801 National Rd W Richmond, IN, EEUU 47374. Email: deibemi@earlham.edu

4 Departamento de Antropología, Universidad de Chile. Ignacio Carrera Pinto 1045, Santiago, CHILE. Email: mur@uchile.cl
Tarragó 1989; Adán et al. 1995; Uribe y Carrasco 1999; Uribe y Adán 2005). Los principales tipos cerámicos del período Intermedio Tardío (Dupont, Ayquina, Rojo Violáceo, Turi Rojo Alisado y sus variantes) se encuentran en una amplia gama de sitios que abarcan el Loa Medio (Lasana y Quillagua), el Loa Superior (Turi, Caspana, Toconce), San Pedro de Atacama (Tarragó 1989; Uribe 2002) y otros lugares lejanos (Núñez et al. 2003; Nielsen et al. 2006; Pimentel et al. 2007; González y Westfall 2008; Pimentel 2009).

Sin embargo, en este detallado conocimiento de la cultura material de estas dos regiones, falta un análisis igual de detallado sobre las relaciones entre las comunidades involucradas que produjeron y utilizaron el componente cerámico Loa-San Pedro. Un intento relativamente reciente por entender arqueológicamente las relaciones prehispánicas entre ambas zonas, corresponde al análisis por activación neutrónica instrumental o AANI aplicado a cerámica del período Tardío de los sitios Turi y Catarpe (Alden et al. 2006). Éste concluyó que la producción cerámica se realizaba en las dos localidades e identificó el posible traslado de materiales desde Turi en el río Salado, a Catarpe en San Pedro. Sobre esta base, los autores plantean que el sitio de Catarpe fue un centro dominante dentro del sistema administrativo incaico (Alden et al. 2006: 589) y fue en ese marco que el sitio consumió cerámica de Turi.

Se presentan, no obstante, una serie de preguntas. ¿Por qué consumir vasijas de los mismos estilos entre comunidades vecinas? ¿Nos enfrentamos tal vez al movimiento de materias primas, artesanos o vasijas? Asimismo, es igualmente factible que las comunidades del río Salado tuvieran un importante centro de producción cerámica y fue por eso que se trasladaron objetos o materias desde allí. Por tanto, Turi también pudo ser un centro dominante y motivó a los habitantes de Catarpe a consumir sus vasijas, materias primas, o trasladar alfareros para emular los estilos del Loa Superior. Quizás no se trató de diferencias de poder, sino de procesos más complejos como aquellos identitarios postulados por investigadores para el período Intermedio Tardío (Agüero et al. 1999; Agüero 2000, 2004, 2012). Esta posibilidad no es menor, ya que Adán y colaboradores (1995: 149) mencionan, por ejemplo, que habitantes de la comunidad de Caspana se distinguen de los del pueblo de Ayquina ya que "[...] no adquieren su cerámica de Toconce, como Ayquina, sino 


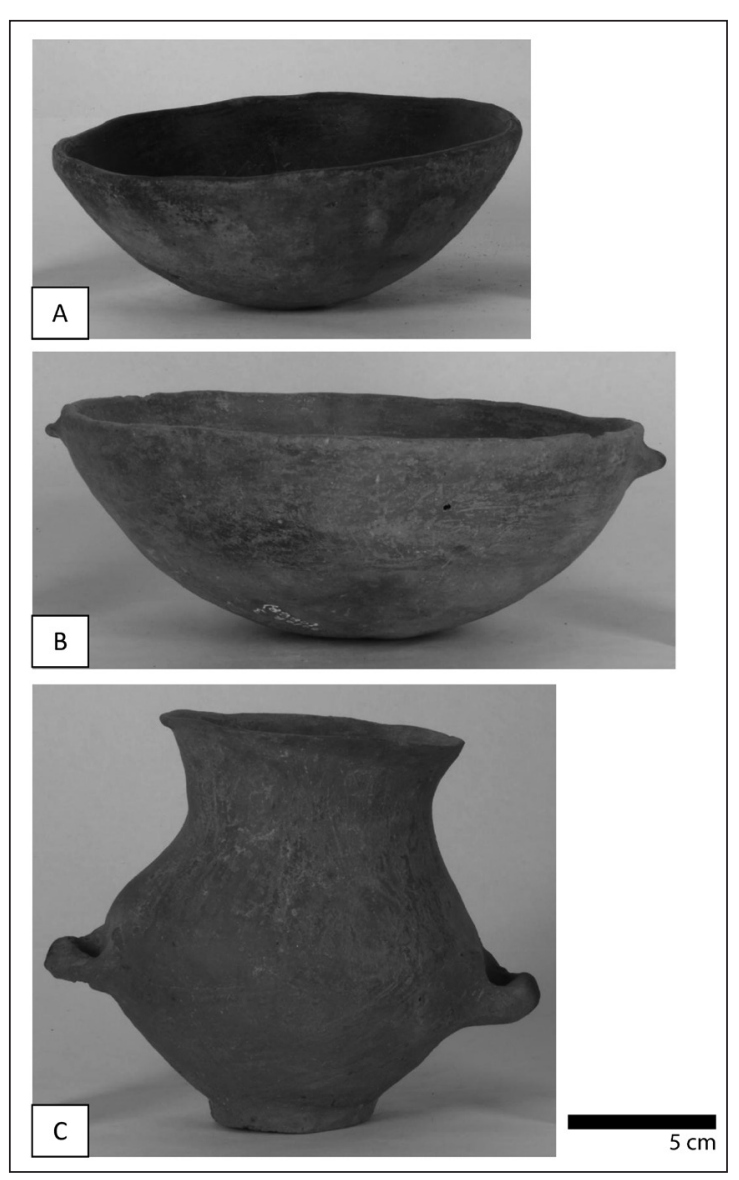

Figura 2. Los estilos cerámicos del período Intermedio Tardío: a) Cerámica Dupont (DUP; no de catálogo 13.290, sitio Coyo 3); b) Cerámica Ayquina (AIQ); c) Cerámica Turi Rojo Revestido (TRR; $\mathrm{n}^{\circ}$ de catálogo 8056, sitio Catarpe). Ejemplares de contextos funerarios. Imágenes reproducidas con permiso del Instituto de Investigaciones Arqueológicas y Museo R.P. Gustavo Le Paige s.j.

de la localidad de Río Grande, la cual se encuentra en la ruta hacia San Pedro de Atacama y conectada con ésta tanto geográfica como culturalmente."

Aunque no tengamos información sobre sitios de producción cerámica en el Loa Superior o de San Pedro, la idea de que algunos centros funcionaron como lugares de difusión de estilos y/o vasijas emblemáticas significa que pudieron jugar un rol significativo en la definición de una identidad compartida y/o en las relaciones sociales que la caracterizaron. En suma, en vez de ser testigos de la competencia entre centros dominados por grupos foráneos, estaríamos frente a un proceso de consolida- ción de una identidad suprarregional, relacionada con el componente Loa-San Pedro que produjo nuevos patrones de producción y consumo de piezas que tuvieron un rol emblemático antes de la llegada de los incas. Aunque Alden y colaboradores (2006) se enfocaron en la cerámica del período Tardío, los estilos estudiados por ellos son diagnósticos de todo el Intermedio Tardío y su muestra posiblemente proviene de contextos de ambos períodos. Lo mismo ya ocurría con la coproducción cerámica del Formativo (Sinclaire et al. 1997), lo que sugiere evidencia de una estructura social entrelazada o interdigitada de larga data, más allá de los procesos ocurridos durante el período Tardío (Schiappacasse et al. 1989).

Por lo tanto, en este trabajo se busca profundizar en los temas referidos, ofreciendo los resultados de un estudio arqueométrico exploratorio de muestras de San Pedro y el Loa Superior, teniendo como hipótesis que los materiales cerámicos reflejan prácticas económicas, sociales e identitarias de mayor extensión en el tiempo y mayor complejidad cultural que las dinámicas políticas establecidas por los incas durante el Tardío.

\section{* El componente cerámico Loa-San Pedro}

El componente Loa-San Pedro (Uribe 1997, 2002) se ha definido a partir de una convergencia estilística del Intermedio Tardío en la producción cerámica de comunidades del Loa Superior y San Pedro de Atacama. La cerámica típica del período incluye formas abiertas y simples, pulidas y a veces revestidas, tales como escudillas acompañadas por jarras y urnas alisadas grandes, en ocasiones cubiertas por un revestimiento rojo y otra por un grueso estuco blanco. Las escudillas Dupont (Figura 2a), de interior negro pulido y exterior alisado, serían más comunes al principio del período (ca. 900 DC). Éstas, sobre todo en San Pedro, aparecen acompañadas por grandes vasijas de diversas morfologías revestidas de un pigmento rojo burdeo, llamadas San Pedro Rojo Violáceo. Las escudillas Ayquina (Figura 2b), similares en su forma y el pulimento a las Dupont pero con la superficie interior café a café rojizo, están presentes en menor cantidad en fechas tempranas, pero superan al estilo Dupont en popularidad hacia el 1200 DC. Esta segunda mitad del período Intermedio Tardío está igualmente caracterizada por la presencia de elementos alfareros altiplánicos 
Emily Stovel, William Whitehead, Michael Deibel, Mauricio Uribe

\begin{tabular}{|c|c|c|c|}
\hline Fechas & Período & Fase & Estilos cerámicos asociados \\
\hline $1500-1200 \mathrm{AC}$ & Transición al Formativo & Tajane & Los Morros \\
\hline $1200-500 \mathrm{AC}$ & \multirow{3}{*}{ Formativo } & Tilocalar & \multirow{3}{*}{$\begin{array}{l}\text { Los Morros, Ay B } \\
\text { Loa Café y Rojo Alisados } \\
\text { Rojo Pulido } \\
\text { Negro Pulido (variantes tempranas) }\end{array}$} \\
\hline $500 \mathrm{AC}-100 \mathrm{DC}$ & & Toconao & \\
\hline $100-400 \mathrm{DC}$ & & Sequitor & \\
\hline $400-700 \mathrm{DC}$ & \multirow[b]{2}{*}{ Medio } & Quitor & \multirow{2}{*}{$\begin{array}{l}\text { Negro Pulido (variantes tardías) } \\
\text { Incisos, Negro y Rojo } \\
\text { Gris Grueso Pulido }\end{array}$} \\
\hline $700-950 \mathrm{DC}$ & & Coyo & \\
\hline 950-1100 DC & \multirow{3}{*}{ Intermedio Tardío } & Yaye/Solor & \multirow{3}{*}{$\begin{array}{l}\text { Gris Café Pulido } \\
\text { Dupont Negro Pulido } \\
\text { Rojo Violáceo } \\
\text { Ayquina Café Rojizo } \\
\text { Variantes Turi Rojo }\end{array}$} \\
\hline 1100-1300 DC & & Turi/Quitor & \\
\hline $1300-1450 \mathrm{DC}$ & & Toconce/Zapar & \\
\hline $1450-1550 \mathrm{DC}$ & Tardío & Catarpe & Lasana Café Rojo Revestido \\
\hline
\end{tabular}

Tabla 1. Cronología de San Pedro de Atacama y estilos cerámicos.

(p.e., tipo Hedionda) ${ }^{5}$, variantes de los jarros Turi (p.e., Alisado, Rojo Revestido, etc.; Figura 2c) y grandes piezas utilitarias (cántaros y ollas). ${ }^{6}$

Esta misma convergencia se ve en la producción de textiles y gorros diagnósticos del período, la que está presente en muchas comunidades de la región atacameña, incluyendo

5 Existe debate sobre la influencia altiplánica en el Loa Superior. En un momento inicial, se vio a toda la región bajo dominio atacameño, y luego bajo control altiplánico (a diferencia de San Pedro; Castro et al. 1984). Actualmente hay una corriente de investigación que, reconociendo diferencias materiales y de comportamiento entre las comunidades de San Pedro y el Alto Loa, destaca las continuidades en cuanto a tejidos y cerámica. Hay falta de acuerdo acerca de restos del altiplano boliviano (aparte de la presencia de cerámica Hedionda y enterramientos en chullpas), y la apariencia de elementos altiplánicos durante la segunda mitad del Intermedio Tardío (Uribe 2002; Uribe y Adán 2003; Uribe et al. 2004; Nielsen y Berberián 2008). Esto no quiere decir que no hubo interacción con comunidades altiplánicas, sino que se trató más bien de un proceso temporal en vez de regional (p.e., hay entierros no-subteráneos y cerámica Hedionda también en San Pedro), y aún no se ha aclarado el carácter de estas relaciones que hasta hace poco se vieron como dos extremos: colonización atacameña o altiplánica. Trabajos con múltiples líneas de evidencia nos han ofrecido visiones bastante complejas con respecto a la identidad de comunidades tanto de San Pedro como del Alto Loa (Knudson y Torres-Rouff 2009; Nado et al. 2012). En este trabajo buscamos ampliar cronológicamente la contribución de Alden y colaboradores (2006) para repensar su hipótesis de asimetría política del período Tardío.

6 Ver imágenes en Agüero y colaboradores (1999) y en Uribe (2002). sitios del Noroeste Argentino y el Loa Inferior (Agüero et al. 1999; Agüero 2000, 2004). La producción de estilos diagnósticos de la zona consumidos por una región más amplia que en períodos anteriores ha llevado a postular la construcción de una identidad regional durante esta etapa, tal vez ligada al colapso de Tiwanaku a fines del período Medio (hacia el 900 DC), o por la llegada de nuevos grupos o influencias del sur boliviano durante la segunda mitad de período, después de 1200 DC (Tabla 1). Las evidencias de este proceso de etnogénesis son las similitudes en la cultura material ofrendada en las tumbas y su presencia, con pequeñas diferencias, en varios asentamientos del norte de Chile. Al mismo tiempo, existen evidencias de semejanzas materiales entre las dos regiones desde períodos anteriores (Sinclaire et al. 1997; Agüero 2004), indicando la continuidad de una coproducción estilística en textiles y cerámica; sugiriendo que el patrón del Intermedio Tardío consiste en la extensión geográfica de una entidad social o étnica diversa debido a cambios políticos internos. Lo anterior concuerda con la posibilidad de una construcción étnica ya que muchas veces los grupos aprovechan materiales de potencial simbólico para representar nuevos lazos e identidades (Jones 1997).

Sin embargo, en el caso de la cerámica de San Pedro y el Loa, a partir del Intermedio Tardío ésta pierde presencia (a veces prominente) en las tumbas, así como su carácter refinado y su variedad de formas y colores. Pierde su supuesto rol diagnóstico para la región (si bien lo tuvo en San Pedro 
de Atacama) que suele identificar a la alfarería Negra Pulida del período Medio. Sería difícil, entonces, pensar en los estilos del Intermedio Tardío, más bien burdos, toscamente pulidos y de carácter doméstico, como emblemáticos de una nueva identidad regional o parte del proceso de la construcción de la misma. Entonces, lo interesante de este caso es la coproducción estilística extendida en el tiempo entre las comunidades de ambas regiones, en combinación con diferencias en el uso de estructuras para enterrar individuos en el Loa Superior y distintos tipos de deformación craneana (Knudson y Torres-Rouff 2009). De todo lo anterior, se desprende la posibilidad de haber impuesto modelos culturales mutua y erróneamente excluyentes, por lo que las relaciones entre las comunidades del Loa Superior y San Pedro de Atacama se han entendido como estructuras sociales a veces muy diferentes (Schiappacasse et al. 1989).

Existe una larga historia de cuestionamientos a la existencia de entidades sociales exclusivas en el presente y en el pasado (p.e., Wolf 1982; Stark 1998), así como al uso de modelos integrados para representar el paisaje prehispánico del centro-sur andino (p.e., Martínez 1998; Núñez y Dillehay 1995). En los últimos 10 años, en el área centro-sur andina hay autores que han pensado de otra manera la formación de entidades sociales en el pasado como culturas, etnias, ayllu (Ávila 2005, 2008, 2009; Scattolin 2006a, 2006b; Páez y Giovannetti 2008). En todos estos casos encontramos estilos compartidos y mezclados en regiones amplias, siguiendo patrones que van mucho más allá de la interacción y, tal vez, constituyen sistemas culturales regionales a distintas escalas de integración. El presente estudio, justamente, busca e intenta una primera aproximación a estas preguntas a través la arqueometría que, muchas veces en combinación con análisis estilísticos, puede brindar información relevante sobre las prácticas sociales y relaciones de producción, más allá de las variables formales y decorativas tan consideradas en los estudios cerámicos.

\section{* Objetivos del estudio}

Para este estudio se utilizó un equipo portátil de fluorescencia de rayos-X (FRXp) y comparamos los componentes químicos elementales y su variación en el caso de tres tipos cerámicos del período Intermedio Tardío. La combinación única de niveles elementales de ciertos tipos cerámicos se ha relacionado con la geología de su lugar de manufactura
(Glascock y Neff 2003), pero el instrumento ocupado aquí no ofrece mayor información sobre posibles fuentes mineralógicas diferenciadas. El uso de FRX portátil ha sido cuestionado en comparación con otros métodos arqueométricos como instrumentos no-móviles de FRX porque sus detectores inadecuados y tiempos de análisis muy cortos no permiten suficiente precisión analítica (Craig et al. 2007; Shackley 2010, 2011; Speakman y Shackley 2013). Estas críticas apuntan a que la falta de estándares certificados impide la comparación de los resultados entre varios instrumentos, impidiendo la confirmación o rechazo de los datos obtenidos con instrumentos portátiles.

Sin embargo, durante más de cinco años, tenemos resultados importantes de investigaciones de obsidiana y cerámica con instrumentos portátiles (Williams-Thorpe et al. 1999; Jia et al. 2010; Forster et al. 2011; Sheppard et al. 2011; Speakman et al. 2011; Goodale et al. 2012; Mitchell et al. 2012; Frahm 2013; Frahm y Doonan 2013; Frahm et al. 2013). Estos estudios mostraron que el FRXp ha podido identificar los mismos agrupamientos que otros métodos (a veces con distintos elementos diagnósticos), y que estos grupos tienen valor arqueológico interpretativo, que la consistencia interna de los instrumentos ofrece un nivel importante de confiabilidad en los datos y, como ya veremos, el uso de estándares demuestra que FRX portátil puede confirmar resultados precisos obtenidos con otros instrumentos. La utilidad primaria del instrumento es la posibilidad de analizar una muestra muy grande sin moverla ni destruirla.

Nosotros empleamos el FRX portátil como un método exploratorio para determinar patrones de semejanza y diferencia que esperamos profundizar en siguientes fases de estudio y con distintos equipos analíticos de mayor precisión. Se pudo aprovechó esta tecnología para analizar una muestra cerámica del Intermedio Tardío de ambas regiones (Loa y San Pedro de Atacama), para luego evaluar si los patrones de coproducción identificados por Alden y colaboradores (2006), reflejaban prácticas de mayor envergadura geográfica y profundidad temporal. Del mismo modo que aquel estudio anterior, se trabajó con muestras de sitios habitacionales y no funerarios, accediendo a ámbitos más cotidianos que rituales. El análisis se orientó de acuerdo a las siguientes preguntas:

1. ¿Se pueden observar diferencias químicas entre los distintos tipos cerámicos del Loa y San Pedro indicando selección de pastas diferentes para distintos estilos? 
2. ¿Se puede observar una clara diferencia química entre las muestras de las dos subregiones indicando distintas comunidades de producción cerámica durante el Intermedio Tardío?

3. Si se identifican patrones semejantes, ¿se puede identificar alguna variación que implique diferencias políticas entre asentamientos y comunidades?

\section{* Selección de la muestra y metodología}

Para desarrollar el análisis retomamos los tipos cerámicos diagnósticos de sitios clave del Intermedio Tardío de las dos subregiones. Se seleccionaron 251 muestras de los tipos Dupont (DUP), Ayquina (AIQ) y variantes del tipo Turi (TUR), (Tabla 2), procedentes de contextos domésticos de San Pedro de Atacama y el Loa Superior, como Tchaputchayna y Solor 13 (Llagostera y Costa 1999; Agüero 2005) y del Pucara de Turi (Aldunate 1991; Varela et al. 1991), respectivamente.

La muestra de San Pedro se obtuvo de dos colecciones provenientes de prospecciones regionales dentro del marco de dos proyectos de investigación anteriores (Llagostera y Costa 1999; Agüero et al. 2003; Agüero 2005; Núñez 2005; Uribe 2006). En definitiva, se analizó una muestra del 100\% de los estilos cerámicos relevantes (DUP, AIQy TURI) de los sitios Tchaputchayna y Solor 13 ( $n=106$ fragmentos). La muestra del Loa Superior proviene de una colección del Pucara de Turi almacenada en la Corporación de Cultura y Turismo de Calama, a partir de la cual se elaboró la primera secuencia para el sitio y una clasificación cerámica que sigue vigente en la zona (Varela et al. 1991; Llagostera y Costa 1999). Se pudo establecer, por ejemplo, una secuencia cronológica de casi 700 años (ca. 900 a 1560 DC), empezando con los tipos Turi Rojo Alisado y Burdo, Ayquina y Dupont, en con-

\begin{tabular}{|c|c|c|c|}
\cline { 2 - 4 } \multicolumn{1}{c|}{} & Cronología (DC) & San Pedro & Río Salado \\
\hline DUP & $900-1200$ & 57 & 45 \\
\hline AIQ & $900-1400$ & 46 & 89 \\
\hline Turi & $900-1400$ & 3 & 11 \\
\hline
\end{tabular}

Tabla 2. Muestra analizada según tipo y subregión. Fuente: Uribe (2002). traste con contextos posteriores que contienen los mismos tipos en menos cantidades y el aumento de variantes Turi Rojo Revestido o Pintado (Aldunate 1991). Como se ha mencionado, la división en dos fases con un momento inicial dominado por Dupont y en menor escala Ayquina y cántaros alisados, seguido por otro posterior caracterizado mayormente por Ayquina y Turi Rojo Revestido en asociación con piezas de tipo altiplánico de Lípez fue confirmado por Uribe (2002). En esta oportunidad se analizó la totalidad de los estilos relevantes de tres cajas seleccionadas de forma aleatoria $(n=145)$.

Este proyecto se ejecutó en junio de 2011 en el laboratorio arqueológico del Instituto de Investigaciones Arqueológicas y Museo R.P. Gustavo le Paige en San Pedro de Atacama, Chile. El instrumento utilizado es un Innov-X Alpha portátil con un ánodo de tungsteno, 10-40 kV, 10-50 $\mu \mathrm{A}$, fuente de rayos-X, detector de diodos de silicio PiN, <230 eV FWHM a $5.95 \mathrm{keV}$ Mn linea K-alpha. El instrumento se operó bajo dos modos: 120 segundos para un análisis básico y 120 segundos para un análisis siguiendo el protocolo para elementos livianos. Los resultados de los dos modos se incorporaron a mano con el software Excel y luego se analizaron con JMP Prolo. Las superficies analizadas fueron, en orden de prioridad: cualquier lugar erosionado con la pasta expuesta, la superficie exterior sin engobe de la base o costado de la vasija, luego cualquier superficie de la vasija (en el caso extremadamente raro de no tener una superficie sin engobe).

Se ejecutaron varias pruebas de confiabilidad durante el proceso de toma de datos, incluyendo análisis frecuentes de estándares (modernos y arqueológicos), para evaluar la precisión y la confiabilidad de los resultados. Cada día se analizaron estándares para monitorear la consistencia interna de los resultados y la precisión de los valores obtenidos. Se emplearon estándares certificados por NIST, el National Institute of Standards and Technology del gobierno estadounidense (www.nist.gov) y un estándar local, un fragmento del sitio arqueológico de Coyo Aldea, San Pedro de Atacama. La Tabla 3 compara los valores de titanio (Ti), hierro $(\mathrm{Fe})$, rubidio $(\mathrm{Rb})$, estroncio $(\mathrm{Sr}), y$ circonio (Zr) para NIST 97a, una arcilla de sílex, y NIST $98 \mathrm{~b}$, una arcilla plástica, con los valores certificados por NIST. Para los elementos de valor diagnóstico para este estudio ( $\mathrm{Ti}, \mathrm{Rb}$, and $\mathrm{Sr}$ ), tenemos un grado de acuerdo muy alto entre valores certificados y valores obtenidos. 


\begin{tabular}{|l|c|c|c|c|c|c|}
\cline { 2 - 7 } \multicolumn{1}{c|}{} & \multicolumn{3}{c|}{ NIST $97 \mathbf{b}$ - arcilla de silex (n=26) } & \multicolumn{2}{c|}{ NIST 98b - arcilla plástica (n=26) } \\
\hline Elemento & $\begin{array}{c}\text { Promedio de } \\
\text { valores obtenidos }\end{array}$ & Valor certificado & $\begin{array}{c}\text { Desviación } \\
\text { estándar }\end{array}$ & $\begin{array}{c}\text { Promedio de } \\
\text { valores obtenidos }\end{array}$ & Valor certificado & $\begin{array}{c}\text { Desviación } \\
\text { estándar }\end{array}$ \\
\hline $\mathrm{Ti}$ & $14400 \pm 300$ & $14300 \pm 400$ & 300 & $7900 \pm 200$ & $8090 \pm 120$ & 200 \\
\hline $\mathrm{Fe}$ & $7400 \pm 200$ & $8310 \pm 80$ & 100 & $10700 \pm 200$ & $11800 \pm 100$ & 100 \\
\hline $\mathrm{Rb}$ & $32 \pm 1$ & 33 (sin certificación) & 1 & $162 \pm 3$ & 180 (sin certificación) & 2 \\
\hline $\mathrm{Sr}$ & $85 \pm 2$ & $84 \pm 2$ & 2 & $186 \pm 4$ & $189 \pm 8$ & 4 \\
\hline $\mathrm{Zr}$ & $408 \pm 8$ & $\begin{array}{c}500(\text { sin } \\
\text { certificación) }\end{array}$ & 7 & $218 \pm 4$ & 220 (sin certificación) & 4 \\
\hline
\end{tabular}

Tabla 3. Resultados del instrumento portátil de FRX comparado con valores certificados para dos estándares del National Institute of Standards and Technology (arcillas plástica y de sílex).

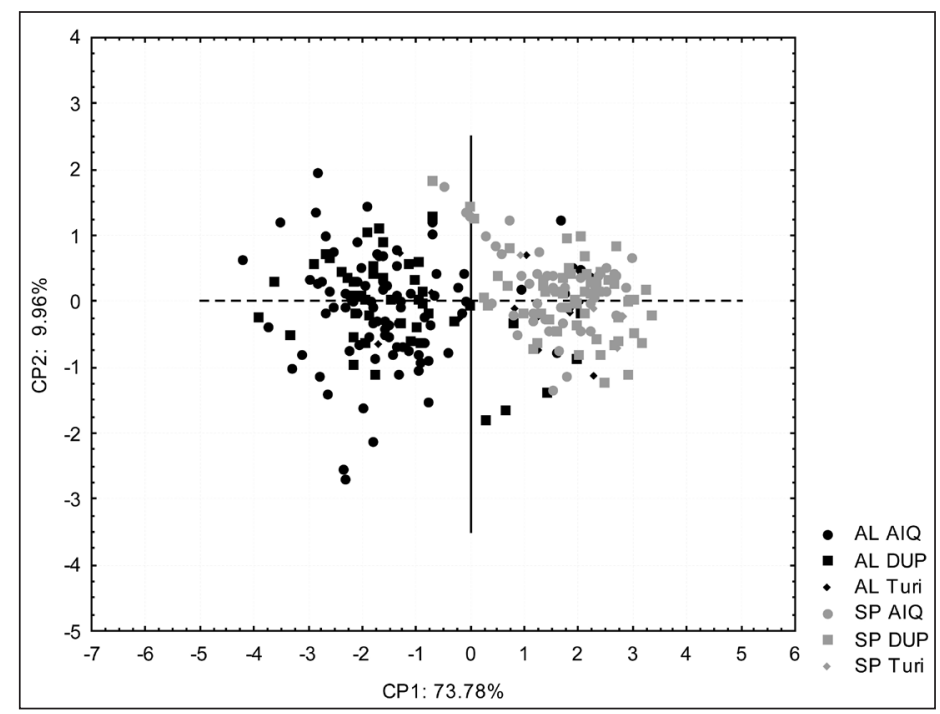

Figura 3. Gráfico de componentes principales, explicando $85 \%$ de la variación en la muestra.

Nuestros valores para Fe y Zr son bajos pero los datos tienen una correlación lineal, lo que permite corregir la diferencia (Craig et al. 2007). No hay tampoco variabilidad en los valores obtenidos en el tiempo (p.e., ausencia de instrument drift; Speakman y Shackley 2013). Mediante lo presentado, tenemos confianza en la confiabilidad y precisión de los datos obtenidos y, por lo tanto, en el modelo presentado en la Tabla 3.

El proceso de reducción de variables (Baxter y Jackson 2001) y limpieza de datos duros incluyó la identificación de nueve elementos ( $\mathrm{Ti}, \mathrm{Mn}, \mathrm{Cr}, \mathrm{Fe}, \mathrm{As}, \mathrm{Rb}, \mathrm{Sr}, \mathrm{Zr}, \mathrm{Pb}$ ) con resultados consistentes para cada muestra, por no estar muy cerca de los limites de detección del instrumento. Cloro y calcio fueron eliminados porque pueden variar debido a procesos tafonómicos postdeposicionales $y$, consecuentemente, no reflejan fielmente la variación asociada a la producción cerámica. En el caso de esos elementos, pueden entrar a la pasta cerámica como sales diluidas en agua, por lo que no los incluimos en nuestros análisis. Se reducen las variables consideradas no solo para eliminar elementos pocos confiables, sino para identificar los elementos diagnósticos, y así facilitar la interpretación geológica de los resultados (Mitchell et al. 2012). Los resultados así reducidos fueron sometidos a análisis de Componentes Principales (Figuras 3 y 4 ) y se 


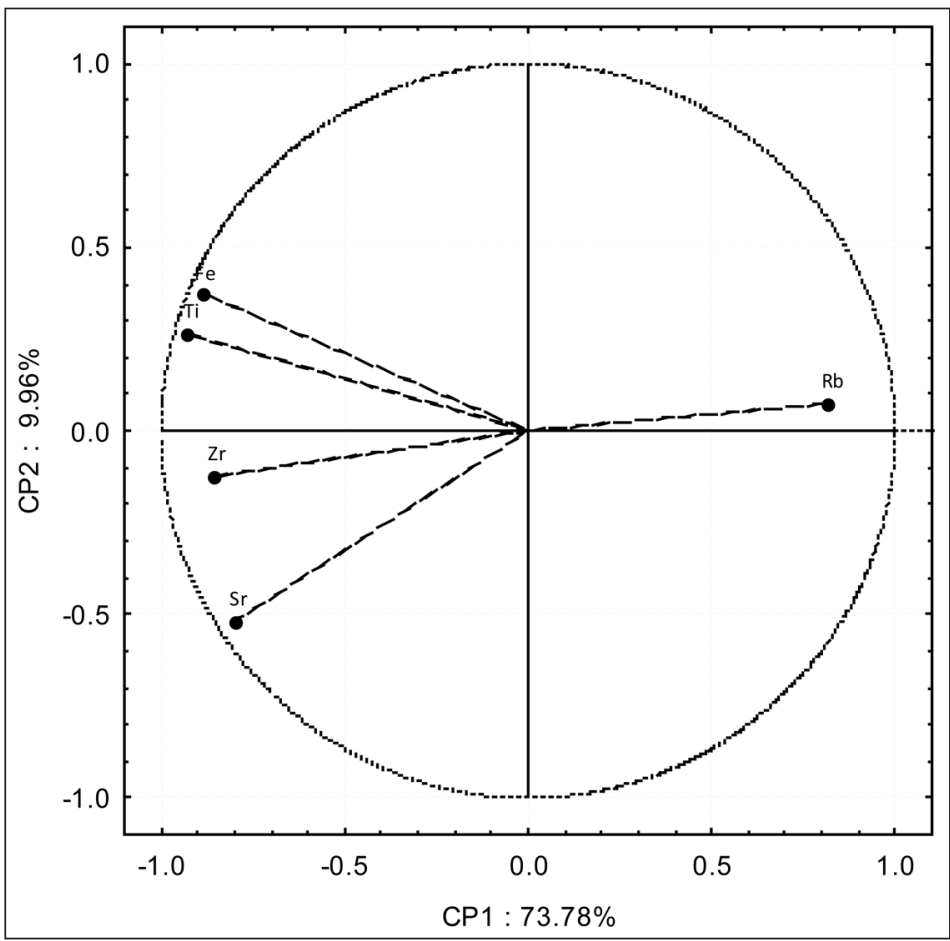

Figura 4. Gráfico de las variables que definen los grupos encontrados en la Figura 2.

\begin{tabular}{|c|c|c|c|c|c|c|c|c|c|c|c|c|c|c|c|c|}
\cline { 2 - 13 } \multicolumn{1}{c|}{} & $\mathbf{K}$ & $\mathbf{C a}$ & $\mathbf{T i}$ & $\mathbf{C r}$ & $\mathbf{M n}$ & $\mathbf{F e}$ & $\mathbf{C o}$ & $\mathbf{C u}$ & $\mathbf{Z n}$ & $\mathbf{A s}$ & $\mathbf{R b}$ & $\mathbf{S r}$ & $\mathrm{Zr}$ & $\mathbf{B a}$ & $\mathrm{Pb}$ & $\mathbf{C l}$ \\
\hline Río Salado AIQ & 32.21 & 18.21 & 14.19 & 21.15 & 25.07 & 11.30 & 22.34 & 45.31 & 46.19 & 39.99 & 22.46 & 19.37 & 12.19 & 22.59 & 19.74 & 200.22 \\
\hline Río Salado DUP & 35.50 & 32.25 & 15.95 & 24.64 & 41.02 & 12.91 & 23.03 & 72.54 & 13.84 & 28.06 & 21.97 & 15.38 & 11.22 & 23.16 & 19.28 & 161.79 \\
\hline San Pedro AIQ & 25.03 & 56.74 & 13.85 & 20.66 & 33.25 & 15.15 & 25.03 & 37.43 & 13.31 & 104.06 & 17.53 & 12.92 & 12.73 & 30.49 & 23.83 & 221.58 \\
\hline San Pedro DUP & 25.83 & 28.23 & 15.83 & 27.40 & 31.34 & 13.09 & 28.25 & 52.74 & 25.15 & 46.91 & 12.63 & 13.53 & 13.12 & 30.55 & 25.28 & 191.92 \\
\hline Río Salado Turi & 35.82 & 85.59 & 16.03 & 32.24 & 21.34 & 11.74 & 26.61 & 37.81 & 22.08 & 19.62 & 23.96 & 15.86 & 12.64 & 13.42 & 13.55 & 19.93 \\
\hline San Pedro Turi & 24.84 & 18.10 & 22.39 & 31.70 & 37.95 & 16.53 & 38.36 & 12.64 & 6.54 & 11.26 & 9.84 & 9.77 & 8.19 & 4.55 & 6.19 & 33.38 \\
\hline
\end{tabular}

Tabla 4. Coeficientes de variación por tipo cerámico.

calcularon coeficientes de variación para comparar los tipos cerámicos estudiados (Tabla 4).

\section{* Resultados}

Los Figuras 3 y 4 incluyen los dos primeros factores del análisis de Componentes Principales y representan más del $80 \%$ de la variación en la muestra. Los resultados demuestran claras diferencias químicas entre la cerámica encontrada en las dos regiones. Hay una cierta cantidad de fragmentos producidos con materiales del conjunto del río Salado encontrado en sitios habitacionales de San Pedro, pero ningún fragmento hecho con materiales con características del grupo de San Pedro en la colección del Loa Superior. El eje de diferenciación entre estos grupos regionales es con respecto a la presencia o ausencia de rubidio (Rb). Se podría hablar, entonces, de un grupo alto 
en contenido de Rb para San Pedro y otro con bajos niveles de $\mathrm{Rb}$ para el Loa Superior.

Por una parte, la cerámica pulida (DUP y AIQ) de contextos domésticos de Turi y San Pedro de Atacama exhibe mucha semejanza en cuanto a sus niveles de homogeneidad. Un análisis por estilo y región (ver Tabla 4) hace evidente que la cerámica de ambas zonas sigue el mismo patrón, salvo en el caso del calcio, zinc y arsénico ( $\mathrm{Ca}, \mathrm{Zn}$ y As) donde el tipo AIQ de San Pedro es más variable que el tipo DUP de San Pedro; AIQ del Loa varía más que DUP del Loa y AIQ de San Pedro es más variable que DUP de San Pedro, respetivamente. Estos casos sugieren una leve diferenciación en la producción cerámica de estos estilos a lo largo del Intermedio Tardío con respecto a estos elementos químicos, ya que los fragmentos AIQ más tardíos suelen ser más variables en esos elementos que DUP, el estilo más temprano. Esta variación composicional puede ser producto de una causa ambiental; es decir, una variación provocada por las materias primas usadas en la manufactura, introducidas durante el proceso de producción o después de la depositación de las piezas (Arnold 2000). Igualmente podría ser producto de diferencias en la adherencia a las normas productivas con respecto a los distintos estilos o con el tiempo.

Por otra parte, la muestra cerámica de San Pedro de Atacama es altamente enriquecida en rubidio, metal alcalino igual que el litio, sodio, potasio, cesio y francio ( $\mathrm{Li}, \mathrm{Na}, \mathrm{K}$, Cs y Fr). Se encuentra en rocas ígneas como el granito, la andesita y el basalto. Las arcillas de San Pedro provienen de componentes erosionados y depositados de esas rocas $y$, por lo tanto, contienen esa alta concentración de rubidio. Debido al mismo proceso de depositación geológica, San Pedro es una de las fuentes de litio más importantes del mundo. Con tal concentración de metales alcalinos en el Salar de Atacama sería lógico encontrar una importante concentración de estos elementos para distinguir las muestras cerámicas de los oasis atacameños, aunque todavía estamos muy limitados en la posibilidad de precisar los niveles de todos estos metales (salvo para el caso del Rb). Las muestras del Loa Superior no se caracterizan por los mismos procesos de depositación y acumulación de metales alcalinos, aunque poseen componentes erosionados del mismo espectro geológico que representan los Andes. Estudios arqueométricos futuros deberán estudiar y comparar otros minerales ricos en rubidio como la moscovita, presentes en los desgrasantes o componentes naturales de las arcillas, lo cual es muy típico en la alfarería del Loa Superior.

\section{* Conclusiones}

Los resultados sugieren que los perfiles químicos de los mismos tipos cerámicos producidos en el Loa Superior y San Pedro de Atacama durante el Intermedio Tardío son muy distintos y que los alfareros de ambas regiones produjeron los estilos característicos del período con sus propios recursos regionales. Eso confirma el patrón identificado por Alden y colaboradores (2006), pero se extiende en el tiempo a todo el Intermedio Tardío y no solo a la época incaica. Estaríamos, por lo tanto, frente a una coproducción de estilos cerámicos de larga data por dos grupos o comunidades distintas e, incluso, es posible un traslado unidireccional de materiales o artesanos desde el río Salado a San Pedro de Atacama. Estos resultados ponen en duda el argumento que ese traslado fue el producto de diferencias políticas entre centros administrativos establecidos por una fuerza foránea como pudieron ser los incas, sino que más bien podrían corresponder a un patrón más generalizado y a una dinámica propia de estas comunidades locales. Esta direccionalidad dataría de inicios del Intermedio Tardío (ca. 900 DC) y, tal vez, refleje una distinción jerárquica entre las regiones del Salar de Atacama y el Loa Superior con respecto a su estatus cultural y como productor de cerámica, siendo este último el más importante, como se observa hasta momentos etnográficos (Varela 2002).

De este modo, nuestro trabajo demuestra la posibilidad de replicar los resultados encontrados por Alden y sus colegas (2006), ya que la comparación con materias primas analizadas por éstos respalda la idea que las diferencias observadas en nuestros resultados tiene que ver con la producción simultánea de los mismos estilos en ambos lugares. Se requiere, sin embargo, la contextualización cronológica de estos resultados con un análisis diacrónico que considere semejanzas y diferencias arqueométricas de la producción alfarera de las dos regiones, así como su yuxtaposición con la cerámica de los sitios funerarios. Queda pendiente la pregunta, si la producción y/o consumo de estos estilos fue el trabajo y la responsabilidad de cada unidad doméstica, ayllu, pueblo, región, o si hubo 
una centralización de la producción para enfrentar tensiones regionales debido a problemas ecológicos, étnicos y/o políticos (Uribe y Adán 2005). En definitiva, sigue habiendo dudas con respecto a la estructura social de todo este conjunto cultural que debe ser explorada arqueológicamente (Pimentel y Montt 2008; Sepúlveda 2011).

A partir de lo anterior, no obstante, es posible hipotetizar la existencia de cierta unidad material durante el Intermedio Tardío en cuanto a estilos cerámicos, textiles y arte rupestre (Uribe 2002; Pimentel y Montt 2008; Agüero 2012), la que a su vez ocultaría importantes diferencias interregionales. Dentro de este sistema cultural la cerámica parece haber jugado un rol particular en el sentido de que, a pesar de pequeñas diferencias regionales en la frecuencia y presencia de ciertos ejemplares (Uribe 2002), los tipos cerámicos encontrados en el Loa no varían en su estilo, como ocurre con los textiles que pueden mostrar elementos híbridos (Agüero et al. 1999; Agüero 2012). Además, su amplia distribución a lo largo de los períodos Intermedio Tardío y Tardío sugiere que, a pesar de ser productos sencillos de uso cotidiano, estos estilos fueron relevantes para una amplia gama de comunidades y que, tal vez, el consumo de ellos tanto en la vida doméstica como en la actividad funeraria, fueron emblemáticos de una identidad suprarregional. Es posible, por lo tanto, que estemos frente a una convergencia productiva de cerámica que sería parte de la construcción de una identidad "atacameña" panregional durante este período (Agüero et al. 1999; Uribe 2002); lo que tendría algunos antecedentes en el período Formativo, puesto que las comunidades del Loa Superior mantuvieron un fuerte contacto con el Salar de Atacama hasta el período Medio (Sinclaire et al. 1997), cuando se produce un quiebre por la influencia Tiwanaku en San Pedro. De la misma manera, ahora la estética cerámica pudo haber adquirido un rol emblemático en esa identidad junto con otras materialidades como los textiles (Agüero 200o). En este sentido, a futuro sería interesante establecer correspondencias químicas entre objetos similares encontrados en lugares como Quillagua (Loa Inferior) y otros puntos del Loa, para períodos contemporáneos y más tempranos.

En suma, en vez de una nueva producción de cerámica con potencial emblemático, proponemos la existencia de una coproducción doméstica de los mismos estilos con distintas fuentes de materias primas a lo largo de toda la época prehispánica tardía de esta parte de la Circumpuna. Esta producción mutua se sitúa dentro de un contexto material y cultural muy complejo de semejanzas y diferencias superpuestas a distintas escalas entre San Pedro y el río Loa, así como con otras comunidades del norte de Chile, sur de Bolivia y norte de Argentina. Recomendamos, por lo tanto, el estudio diacrónico, sistemático y de múltiples líneas de evidencia de las expresiones materiales compartidas y diferenciadas de las comunidades centro-sur andinas. Esto contribuirá a entender los complejos sistemas que establecieron estos heterogéneos grupos dentro de una comunidad regional, sin necesidad de recurrir a explicaciones fundamentadas en agentes externos que no permiten valorar las dinámicas andinas más locales.

Agradecimientos Una versión resumida de este trabajo fue presentada en el Tercer Congreso de Arqueometría Latinoamericana en Arica, noviembre de 2011. Esta investigación fue posible gracias al apoyo de la Vicerrectoría de Investigación y Desarrollo Tecnológico de la Universidad Católica de Norte (VRIDT 162/2011) y Earlham College. Agradecemos el apoyo de Mario Pereira, Mark Hubbe, Manuel Arturo Torres, del IIAM-UCN, a Eileen y Michael Beard, a los funcionarios de Dumbarton Oaks, a la Corporación de Cultura y Turismo de Calama y, por supuesto, a los evaluadores del manuscrito.

\section{$*$ Referencias citadas}

ADÁN, L. y M. URIBE, 2005. El dominio inca en la localidad de Caspana: Un acercamiento al pensamiento político andino (río Loa, norte de Chile). Estudios Atacameños 29: 41-66.
ADÁN, L., M. URIBE, P. ALLIENDE y N. HERMOSILLA, 1995. Entre el Loa y San Pedro: Nuevas investigaciones arqueológicas en Caspana. Hombre y Desierto 9(2): 147-155. 
AGÜERO, C., 2000. Fragmentos para armar un territorio: La textilería en Atacama durante los períodos Intermedio Tardío y Tardío. Estudios Atacameños 20: 7-28.

2004. Componente Tiwanaku vs. componente local: El período Medio en los oasis de San Pedro de Atacama. En Tejiendo sueños en el Cono Sur. Textiles andinos: Pasado, presente y futuro, V. Solanilla (Ed.), pp. 180-198. Grup d'Estudis Precolombins, Universitat Autonoma de Barcelona, Barcelona.

2005. Aproximación al asentamiento humano temprano en los oasis de San Pedro de Atacama. Estudios Atacameños 30: 2960.

2012. El rol del vestuario en la sociedad Pica-Tarapaca (800-1300 DC). Arqueología textil del norte de Chile, Andes del Sur. Editorial Académica Española, Saarbrücken. https://www.morebooks. de/store/es/book/el-rol-del-vestuario-en-la-sociedad-picatarapac\%C3\%A1-800-1300-d-c/isbn/978-3-8484-6099-1

AGÜERO, C., L. NÚÑEZ, M. URIBE y C. CARRASCO, 2003. Registro arqueológico y cronología del período Formativo en los oasis de San Pedro de Atacama. Boletín de la Sociedad Chilena de Arqueología 35:3-4.

AGÜERO, C., M. URIBE, P. AYALAy B. CASES, 1999. Una aproximación arqueológica a la etnicidad y el rol de los textiles en la construcción de la identidad cultural en los cementerios de Quillagua. Gaceta Arqueológica Andina 25: 167-198.

ALDEN, J., L. MINC y T. LYNCH, 2006. Identifying the sources of Inka period ceramics from northern Chile: Results of a neutron activation study. Journal of Archaeological Science 33 (4): 575-594.

ALDUNATE, C., 1991. Arqueología en el Pukara de Turi. Boletín del Museo Regional de La Araucanía 4(2): 61-78.

ARNOLD, D., 2000. Does the standardization of ceramic pastes really mean specialization? Journal of Archaeological Method and Theory 7(4): 333-375.

ÁVILA, F., 2005. El estilo alfarero yavi y su relación con la construcción de entidades culturales. Teoría 1: 85-101.

2008. Un universo de formas, colores y pinturas: Caracterización del estilo alfarero yavi de la puna nororiental de Jujuy. Intersecciones en Antropología 9: 197-212.

2009. Interactuando desde el estilo. Variaciones en la circulación espacial y temporal del estilo alfarero yavi. Estudios Atacameños, Arqueología y Antropología Surandinas 37: 29-50.
BAXTER, M. y C. JACKSON, 2001. Variable selection in artefact compositional studies. Archaeometry 43(2): 253-268.

BERENGUER, J., 1998. La iconografía del poder en Tiwanaku y su rol en la integración de zonas de frontera. Boletín del Museo Chileno de Arte Precolombino 7: 19-37.

1978. La problemática Tiwanaku en Chile: Visión retrospectiva. Revista Chilena de Antropología 1: 17-40.

BERENGUER, J. y P. DAUELSBERG, 1989. El norte grande en la órbita de Tiwanaku (400 a 1200 DC). En Culturas de Chile. Prehistoria: Desde sus orígenes hasta los albores de la Conquista, J. Hidalgo, V. Schiappacasse, H. Niemeyer, C. Aldunate e I. Solimano (Eds.), pp. 129-180. Editorial Andrés Bello, Santiago.

BERENGUER, J., I. CÁCERES, C. SANHUEZA y P. HERNÁNDEZ, 2005. El Qhapaqñan en el Alto Loa, norte de Chile: Un estudio micro y macromorfológico. Estudios Atacameños 29: 7-39.

BERENGUER, J., V. CASTRO y O. SILVA, 1980. Reflexiones acerca de la presencia de Tiwanaku en el norte de Chile. Estudios Arqueológicos 5: 81-93.

CARRASCO, C., C. AGÜERO, P. AYALA, M. URIBE y B. CASES, 2003. Investigaciones en Quillagua: Difusión del conocimiento arqueológico y protección del patrimonio cultural. Chungara 35: 321-326.

CASTRO, V., 1992. Nuevos registros de la presencia Inka en la provincia de El Loa, Chile. GacetaArqueológica Andina 4(21): 139-154.

CASTRO, V., C. ALDUNATE y J. BERENGUER, 1984. Orígenes altiplánicos de la fase Toconce. Estudios Atacameños 7: 209-235

CASTRO, V., F. MALDONADO y M. VÁSQUEZ, 1991. Arquitectura del "Pukara" de Turi. Boletín del Museo Regional de la Araucanía 4(2): 79-102.

COSTA, M. A., W. NEVES y M. HUBBE, 2004. Influencia de Tiwanaku en la calidad de vida biológica de la población prehistórica de San Pedro de Atacama. Estudios Atacameños 27: 103-116.

CRAIG, N., R. SPEAKMAN, R. POPELKA-FILCOFF, M. GLASCOCK, J. ROBERTSON, M. SHACKLEY y M. ALDENDERFER, 2007. Comparison of XRF and PXRF for analysis of archaeological obsidian from southern Perú. Journal of Archaeological Science 34(12): 2012-2024.

FORSTER, N., P. GRAVE, N. VICKERY y L. KEALHOFER, 2011. Non-destructive analysis using PXRF: Methodology and application to archaeological ceramics. X-Ray Spectrom 40 (5):389-398. 
FRAHM, E., 2013. Validity of "off the shelf" handheld portable XRF for sourcing Near Eastern obsidian chip debris. Journal of Archaeological Science 40(2): 1080-1092.

FRAHM, E. y R. DOONAN, 2013. The technological versus methodology revolution of portable XRF in archaeology. Journal of Archaeological Science 40(2): 1425-1434.

FRAHM, E., R. DOONAN y V. KILIKOGLOU, 2013. Handheld portable x-ray fluorescence of Aegean obsidians. Archaeometry doi: 10.1111/arcm.12012.

GALLARDO, F., M. URIBE y P. AYALA, 1995. Arquitectura inka y poder en el Pukara de Turi, norte de Chile. Gaceta Arqueológica Andina 24: 151-171.

GLASCOCK, M. y H. NEFF, 2003. Neutron activation analysis and provenance research in archaeology. Measurement Science and Technology 14:1516-1526.

GOODAle, N., D. BAiley, G. JONES, C. PRESCOTT, E SCHOLZ, N. STAGLIANO y C. LEWIS, 2012. pXRF: A study of inter-instrument performance. Journal of Archaeological Science 29: $875-883$.

GONZÁLEZ, C. y C. WESTFALL, 2008. Atacameños en El Salvador: Nuevas apreciaciones sobre un fardo funerario del Cementerio Las Turquesas. Estudios Atacameños, Arqueología y Antropología Surandinas 35: 49-73.

JIA, P., T. DOELMAN, C. CHEN, H. ZHAO, S. LIN, R. TORRENCE y M. GLASCOCK, 2010. Moving sources: A preliminary study of volcanic glass artifact distributions in northeast China using PXRF. Journal of Archaeological Science 37(7): 1670-1677.

JONES, S., 1997. The archaeology of ethnicity: Constructing identities in the past and present. Routledge, Londres y Nueva York.

KNUDSON, K., 2007. La influencia de Tiwanaku en San Pedro de Atacama: Una investigación utilizando el análisis de isótopos de estroncio. Estudios Atacameños 33: 7-24.

KNUDSON, K., y C. TORRES-ROUFF, 2009. Investigating cultural heterogeneity in San Pedro de Atacama, northern Chile, through biogeochemistry and bioarchaeology. American Journal of Physical Anthropology 138(4): 473-485.

LECHTMAN, H., y A. MACFARLANE, 2005. La metalurgia del bronce en los Andes Sur Centrales: Tiwanaku y San Pedro de Atacama. Estudios Atacameños 30: 7-27.

LLAGOSTERA, A. y M. A. COSTA, 1999. Patrones de asentamiento en la época agroalfarera de San Pedro de Atacama (norte de Chile). Estudios Atacameños 17: 175-206.
MARTÍNEZ, J. L., 1998. Pueblos del chañary el algarrobo: Los atacamas en el siglo XVII. DIBAM, Santiago.

NADO, K., S. MARSTELLER, L. KING, B. DAVERMAN, C. TORRES-ROUFF y K. KNUDSON, 2012. Examinando las identidades sociales locales a través de los patrones de variación biológica y cultural en el ayllu de Solcor, San Pedro de Atacama, Chile. Chungara, Revista de Antropología Chilena 44(2):341-357.

NIELSEN, A. y E. BERBERIÁN, 2008. El Señorío Mallku revisitado: Aportes al conocimiento de la historia prehispánica tardía de Lípez (Potosí, Bolivia). En Arqueología de las tierras altas, valles interandinos y tierras bajas de Bolivia. Memorias del I Congreso de Arqueología de Bolivia, C. Rivera (Ed.), pp. 145-166. IIAA-UMSA, PIEB y ASDI/SAREC, La Paz.

NIELSEN, A., J. BERENGUER y C. SANHUEZA, 2006. El Qhapaqñan entre Atacama y Lípez. Intersecciones en Antropología 7: 217-234.

MITCHELL, D., P. GRAVE, M. MACCHERONI y E. GELMAN, 2012. Geochemical characterization of north Asian glazed stonewares: A comparative analysis of NAA, ICP-OES and non-destructive pXRF. Journal of Archaeological Science 39(9): 2921-2933.

NÚÑEZ, L., 2005. La naturaleza de la expansión aldeana durante el Formativo Tardío en la Cuenca de Atacama. Chungara, Revista de Antropología Chilena 37(2): 165-193.

NÚÑEZ, L., C. AGÜERO, B. CASES y P. DE SOUZA, 2003. El campamento minero Chuquicamata 2 y la explotación cuprífera prehispánica en el desierto de Atacama. Estudios Atacameños 25: $7-34$.

NÚÑEZ, L. y T. DILLEHAY, 1995. Movilidad giratoria, armonía social y desarrollo en los Andes Meridionales: Patrones de tráfico e interacción económica. Universidad Católica del Norte, Antofagasta.

PÁEZ, M. y M. GIOVANNETTI, 2008. Tipologizando identidades: Reflexiones sobre la construcción de identidades étnicas en la arqueología del N.O.A.Avá 13: 1-1.

PIMENTEL, G., 2009. Las huacas del tráfico. Arquitectura ceremonial en rutas prehispánicas del desierto de Atacama. Boletín del Museo Chileno de Arte Precolombino 14: 9-38.

PIMENTEL, G. e I. MONTT, 2008. Tarapacá en Atacama. Arte rupestre y relaciones intersocietales entre el 900 y 1450 DC. Boletín del Museo Chileno de Arte Precolombino 13: 35-50.

PIMENTEL, G., I. MONTT, J. BLANCO y A. REYES, 2007. Infraestructura y prácticas de movilidad en una ruta que conectó 
el altiplano boliviano con San Pedro de Atacama. En Procesos sociales prehispánicos en los Andes Meridionales, A. Nielsen, M. C. Rivolta, V. Seldes, M. Vázquez y P. Mercolli (Eds.), pp. 351-382. Editorial Brujas, Córdoba.

OAKLAND, A., 1992. Textiles and ethnicity: Tiwanaku in San Pedro de Atacama, North Chile. Latin American Antiquity 3(4): 316-340.

SCATTOLIN, C., 2006a Categoremas indígenas y designaciones arqueológicas en el Noroeste Argentino prehispánico. Chungara, Revista de Antropología Chilena 38(2): 185-196.

2006b. Contornos y confines del período Formativo en el Noroeste Argentino: El universo iconográfico pre-calchaquí en el valle de Santa María. Estudios Atacameños 32: 119-139.

SCHIAPPACASSE, V., V. CASTRO y H. NIEMEYER, 1989. Los Desarrollos Regionales en el Norte Grande. En Culturas de Chile. Prehistoria. Desde sus orígenes a los albores de la Conquista, J. Hidalgo, V. Schiappacasse, H. Niemeyer, C. Aldunate e I. Solimano (Eds.), pp. 181-226. Editorial Andrés Bello, Santiago.

SEPÚLVEDA, M., 2011. Arte rupestre y complejidad social durante el período Intermedio Tardío en la subregión del río Salado (norte de Chile). Chungara, Revista de Antropología Chilena 43(1): 53-72.

SHACKLEY, M., 2010. Is there reliability and validity in portable $\mathrm{x}$-ray fluorescence spectrometry (PXRF)? The SAA Archaeological Record 10(5): 17-20.

(Ed.), 2011. X-ray fluorescence spectrometry (XRF) in geoarchaeology. Springer, Nueva York.

SHEPPARD, P., G. IRWIN, S. LIN y C. MCCAFFREY, 2011. Characterization of New Zealand obsidian using PXRF. Journal of Archaeological Science 38(1): 45-56.

SINCLAIRE, C., M. URIBE, P. AYALA y J. GONZÁLEZ, 1997. La alfarería del período Formativo en la región del Loa Superior: Sistematización y tipología. Contribución Arqueológica 5: 285-314.

SPEAKMAN, R., N. LITTLE, D. CREEL, M. MILLER y J. IÑANEZ, 2011. Sourcing ceramics with portable XRF spectrometers? A comparison with INAA using Mimbres pottery from the American Southwest. Journal of Archaeological Sciences 38(12): 3483-3496.

SPEAKMAN, R. y M. SHACKLEY, 2013. Silo science and portable XRF in archaeology: Response to Frahm. Journal of Archaeological Science 40(2): 1435-1443.

STARK, M., 1998. The archaeology of social boundaries. Smithsonian Institution Press, Washington D.C.
TARRAGÓ, M., 1989. Contribución al conocimiento arqueológico de las poblaciones de los oasis de San Pedro de Atacama en relación con otros pueblos puneños, en especial el sector septentrional del valle Calchaquí. Tesis doctoral, Universidad Nacional de Rosario, Rosario.

TORRES-ROUFF, C., 2002. Cranial vault modification and ethnicity in Middle Horizon in San Pedro de Atacama, Chile. Current Anthropology 43(1): 163-171.

2007. La deformación craneana en San Pedro de Atacama. Estudios Atacameños 33: 25-38.

TORRES-ROUFF, C., M. A. COSTA y A. LLAGOSTERA, 2005. Violence in times of changes: The Late Intermediate period in San Pedro de Atacama. Chungara, Revista de Antropología Chilena $37(1): 75-83$.

URIBE, M., 1997. La alfarería de Caspana y su relación con la prehistoria tardía de la subárea Circumpuneña. Estudios Atacameños 17: $243-262$.

2002. Sobre alfarería, cementerios, fases y procesos durante la prehistoria tardía del desierto de Atacama (800-1600 DC). Estudios Atacameños 22: 7-32.

2004. El Inka y el poder como problemas de la arqueología del Norte Grande de Chile. Chungara, Revista de Antropología Chilena 36:313-324.

2006. Sobre cerámica, su origen y complejidad social en los andes del desierto de Atacama, norte de Chile. En Esferas de interacción prehistóricas y fronteras nacionales modernas: Los Andes sur centrales, H. Lecthman (Ed.), pp. 449-502. Instituto de Estudios Peruanos (IEP), Lima.

URIBE, M. y L. ADÁN, 2003. Arqueología, poblaciones originarias y patrimonio cultural en el desierto de Atacama. Chungara $35: 295-304$.

2005. Arqueología, historia cultural y evolución social en el desierto de Atacama (900-1700 DC). Actas del XVI Congreso Nacional de Arqueología Chilena, pp. 263-274. Arica.

URIBE, M. y C. AGÜERO, 2001. Alfarería, textiles y la integración del Norte Grande de Chile a Tiwanaku. Boletín de Arqueología PUCP 5:397-426.

2004. Iconografía, alfarería y textilería Tiwanaku: Elementos para una revisión del período Medio en el Norte Grande de Chile. Chungara, Revista de Antropología Chilena 36(2): 10551068. 
URIBE, M., L. ADÁN y C. AGÜERO, 2002. El dominio inka, identidad local y complejidad social en las tierras altas del desierto de Atacama, Norte Grande de Chile (1450-1541 DC). Boletín de Arqueología PUCP 6:301-336.

2004. Arqueología de los períodos Intermedio Tardío y Tardío de San Pedro de Atacama y su relación con la cuenca del río Loa. Chungara, Revista de Antropología Chilena 36(2): 943-956.

URIBE, M. y C. CARRASCO, 1999. Tiestos y piedras talladas: La producción cerámica y lítica en el período Tardío del Loa Superior. Estudios Atacameños 18: 55-72.

VARELA, V., 1999. El camino del Inka en la cuenca superior del río Loa, desierto de Atacama, norte de Chile. Estudios Atacameños 18: 89-105.
2002. Enseñanzas de alfareros toconceños: Tradición y tecnología en la cerámica. Chungara 34: 225-252.

VARELA, V., M. URIBE y L. ADÁN, 1991. La cerámica arqueológica del sitio Pukara de Turi: O2-Tu-002. Boletín del Museo Regional de LaAraucanía 4(II): 107-121.

WILLIAMS-THORPE, O., P. POTTS y P. WEBB, 1999. Field-portable non-destructive analysis of lithic archaeological samples by X-ray fluorescence instrumentation using a mercury iodide detector: Comparison with wavelength-dispersive XRF and a case study in British stone axe provenancing. Journal of Archaeological Science 26(2): 215-237.

WOLF, E., 1982. Europe and the people without History. University of California Press, Berkeley. 